\title{
A SYNERGISTIC APPROACH FOR DEEP LEARNING AND KNOWLEDGE ENGINEERED SOLUTIONS
}

\author{
Joshua Haley \\ Richard Pazda \\ Ross Hoehn \\ Robert Wray \\ Soar Technology Inc. \\ Intelligent Systems Division \\ 12124 High Tech Ave, Suite 350 \\ Orlando, FL, USA \\ \{joshua.haley, richard.pazda, ross.hoehn,wray\}@soartech.com
}

\begin{abstract}
Machine Learning techniques offer innovative solutions to many problems from image recognition to intractably difficult games (i.e., Go). However, researchers and developers often employ machine learning techniques in isolation from knowledge-engineered approaches, typically considered passe. While knowledge-engineered solutions have limitations, they offer valuable insights about the problem space and solution pathways. Herein, we explore a hybrid approach that synergistically leverages both knowledge engineering and machine learning.

The mismatch between representational layers of machine-learned systems and explicit, symbolic knowledge in engineered solutions make a hybrid approach appear infeasible. We describe a system of complementary techniques either increase system performance or decrease system implementation cost without reconceptualizing seeming incompatible strategies. This approach applies to problems wherein knowledgeengineered solution can simplify learned information, while a machine learning method can expand problem space boundaries for knowledge-engineered solutions. We illustrate this hybrid approach in Sudoku, enabling empirical assessment of engineering vs. machine learning trade-offs.
\end{abstract}

Keywords: Machine Learning, Deep Learning, Knowledge Engineering, Combined Approaches

\section{INTRODUCTION}

In the last decade, the internet has enabled significant technical advances in both machine learning and data availability that have revolutionized AI development and applications (e.g., Mnih et al. 2015; Mikolov et al. 2013). As a result, many sub-fields of AI - including speech recognition, natural language understanding, robotic path planning, and computer vision - have undergone tremendous re-conceptualizations. While there is on-going debate concerning the theoretical impacts of these innovations (Darwiche 2018), machine learning — specifically deep learning —- has radically transformed the application of AI to real-world problems. 
Although these new methods enable functional capabilities that were not feasible just a few years ago, they have also introduced new costs and considerations during application. A knowledge-engineered system can generate behaviors and produce results that are both human-like and human-interpretable (Feigenbaum 1984, Schreiber, Schreiber, Akkermans, Anjewierden, Shadbolt, de Hoog, Van de Velde, Shadbolt, and Wielinga 2000). Deep learning (DL) does not necessarily deliver either interpretability or human-like performance, which has led to new research exploring methods to regain these capabilities (e.g., explainable AI). DL also generally requires massive sources of data (LeCun, Bengio, and Hinton 2015), which has resulted in attempts to generate realistic, synthetic data when real-world data is unavailable at scale (Sun, Cuesta-Infante, and Veeramachaneni 2019). Finally, a long-recognized limitation of knowledge-engineered (KE) systems is development cost. DL systems are not necessarily more cost-efficient than KE systems, although they do shift the cost from human labor to massive computation (Strubell, Ganesh, and McCallum 2019). However, DL does mitigate the infamous "knowledge acquisition bottleneck" (Feigenbaum 1984) by parallelizing search for data-driven, rather than analytic, solutions.

As applied researchers developing AI, we are interested in developing and applying the best tools to address specific problems. This paper summarizes initial work in exploring various trade-offs and benefits for a combined KE-DL approach. The motivating hypothesis is that a combination of KE and DL may provide a better practical solution than the use of either method in isolation. This combination may be especially apt when the solution quality depends not only on the result or "output," but also on the aforementioned desiderata. We present a systematic exploration of the capabilities resulting from a straightforward combination of KE and DL methods when applied to Sudoku. As we discuss below, Sudoku may superficially seem to be a simple problem, yet general and complete Sudoku solvers present non-trivial challenges for both KE and DL methods.

We have developed a minimally-engineered hybridization of these methods that employs a small hand-coded rule base to an individual puzzle followed by a DL puzzle solver that attempts to complete —- or "fill in" cell values. We vary both the number of a priori rules available and the number of training epochs for ML and then evaluate the relative performance of each method. More sophisticated and complex hybridizations can offer increased utility, such as applying DL only to instances that are difficult to encode via KE. Or conversely, using $\mathrm{KE}$ approaches for data sparse cases or rare events that present training difficultie for DL systems. However, the intent in this work was to explore how fruitfully the two methods could be combined "out of the box" (i.e., without requiring further domain analysis or re-implementation to enable compatible usage). These results illustrate a synergistic effect; the combination of methods outperforms either of the methods used individually. These results are suggestive of the feasibility of developing simple, cheap, incomplete knowledge-engineered systems that are extensible, refinable, and made complete via the use of deep learning techniques.

\section{SUDOKU DOMAIN}

A Sudoku is a puzzle embedded onto an $n \times n$ grid (typical $n=9$ ); the objective is to populate all unoccupied spaces within the grid such that each column, row and 3x3 sub-grid contains the integer values 1-9 exactly once (Delahaye 2006). A well-formed Sudoku puzzle (Figure 1), presents a partially complete grid that has exactly one correct solution. Well-formed Sudoku puzzles require only deductive logic to derive their solution (i.e., guessing is not required). There are $6.67 \times 10^{21}$ unique completed puzzles (Felgenhauer and Jarvis 2006) and 5.5B essentially different completed grids (Russell and Jarvis 2006). The number of unique grids presents a particular challenge for a machine learning approach: the system will likely need to discover rotations and other permutations to reduce the size of the solution space. KE approaches, which typically adopt a constraint-based representation (rather than knowledge specific to cell location), would treat nonessentially different grids the same. For example, the "tactics" we describe below encode methods for cell completion that apply to rows or columns without distinction. 


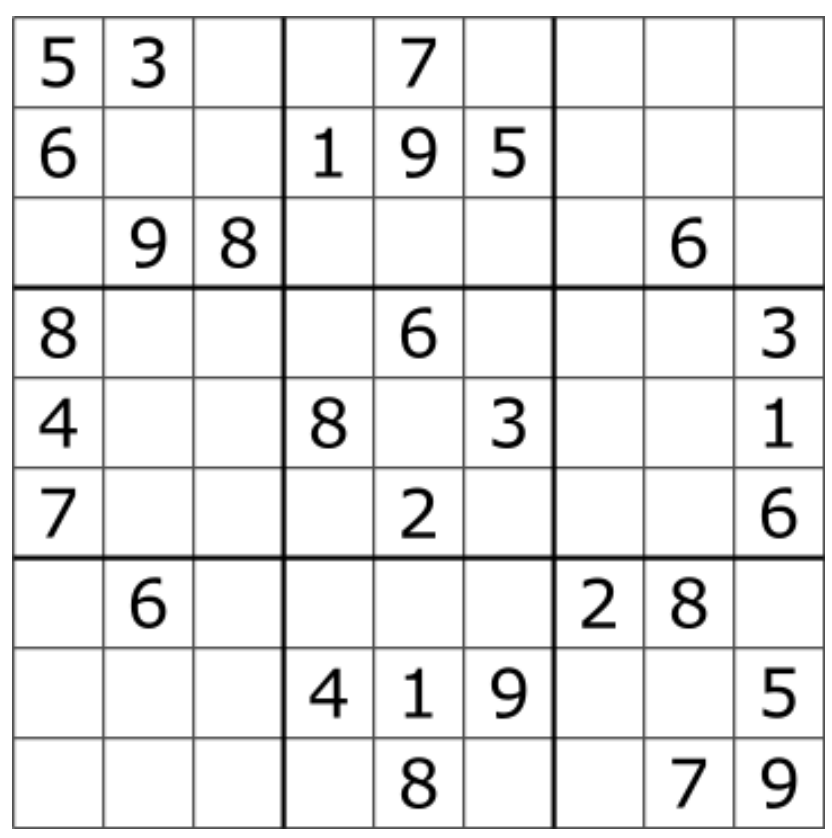

Figure 1: Typical Sudoku Puzzle.

General solution methods for Sudoku puzzles are NP-complete and equivalent to Boolean satisfiability (SAT) problems (Lynce and Ouaknine 2006). While present computing technologies are capable of employing simple search methods to search a 9x9 grid exhaustively, we are interested in solutions that mimic the solving process used by human solvers; human solvers generally utilize rules and game constraints to solve a puzzle without guessing (Delahaye 2006). Competing constraints lead to several reoccurring patterns and multi-step logical inferences that are used to solve the puzzle incrementally.

In simpler puzzles, a human solver can use direct exclusion and inclusion "tactics" to solve a grid by determining allowed values for a cell (Lee, Goodwin, and Johnson-Laird 2008). However, human solvers must develop more sophisticated tactics to solve more challenging puzzles. These more complex tactics require simultaneously examining an ever-larger number of cells and the rows, columns, and sub-grids while considering possibly excluded integers and their implications across the puzzle (Lee, Goodwin, and Johnson-Laird 2008). While simple tactics are few and easily encoded, there are many possible advanced tactics. To our knowledge, no one has identified a set of tactics (the deductive rules used by human players) that are guaranteed to solve all Sudoku puzzles. That is, even after more than a decade of academic interest in Sudoku, there does not exist a provably-complete set of tactics capable of solving any Sudoku.

\section{METHOD}

These properties of Sudoku inform the methodology. We implement an incomplete knowledge base of tactics roughly ordered according to the estimated complexity of each tactic to humans (Lee, Goodwin, and Johnson-Laird 2008). The rule-based representation of the tactics allows for direct control/variation of the tactics available to the solver for any single puzzle.

We use a DL model trained to complete puzzles that are not fully solved after employing KE tactics. Conceptually, we use the available KE tactics to simplify the ML problem, followed by ML to both enhance and extend the incomplete tactical knowledge-base. This hybrid approach expands the range of problems the overall system can solve. This method is similar to the works of (Wolfman and Weld 2001) in that the KE represents a principled approach to solving the underlying SAT problem, while the DL provides a data 
Table 1: Examples of "Simple" and "Advanced" Tactics for Rows/Columns/Boxes (R/C/B).

\begin{tabular}{cll} 
\# & Label & Description \\
\hline \hline & Simple Tactics & \\
\hline 1 & Remove Candidate & $\begin{array}{l}\text { If candidate for cell has already been filled in R/C/B, remove it as a candidate for } \\
\text { cell }\end{array}$ \\
2 & Complete Singles & If a candidate appears in only cell in any R/C/B, fill the cell with the candidate \\
\hline \hline & Advanced Tactics & \\
\hline 6 & Single Pairs & $\begin{array}{l}\text { If pair of cells in a R/C/B that contain only a matching pair of candidate values, } \\
\text { those values can be removed from all other cells in the R/C/B. } \\
\text { If pair of cells in } \mathrm{R} / \mathrm{C} / \mathrm{B} \text { that are the only ones containing the elements of that pair, } \\
\text { all other candidates may be removed. }\end{array}$ \\
\hline
\end{tabular}

influenced heuristic. The general pattern being, use explicit knowledge approaches to simplify, and then principled heuristics when the limits of the encoded knowledge are reached.

The remainder of this section describes the design of both KE and the ML components in greater detail and how each component works together to solve Sudoku puzzle.

\subsection{Tactics-based Solver}

The tactics-based solver encodes a small number of tactics for solving Sudoku. We encode the tactics as individual rules in a production system; one tactic corresponds to a single production rule. The tactics generate "candidates" completions for a cell. Taking the puzzle in Figure 1 as an example, if we iteratively apply Rule 1 of Table 1 to the upper-rightmost cell, we can reduce the candidate integers from (1..9) to $(2,4,8)$. Similarly, application of Rule 2 would allow "8" to be filled in for the middle cell in the lower-left box, as "8" appears as a candidate integer only for that cell within the sub-grid.

These simple tactics are sufficient for solving easier puzzles. More complex combinations of cells, rows, columns, and sub-grids are required to solve some puzzles. Table 1 summarizes two examples of more complex tactics used in this exploration.

As suggested by previous studies, humans solving Sudoku display the ability to deduce tactics for solving Sudoku, even without training and across cultures (Lee, Goodwin, and Johnson-Laird 2008). Human solvers generally demonstrate "simple tactics" initially (using the terminology of Lee, et al. 2008) and then gradually develop more advanced tactics after developing experience at solving puzzles. We implemented 14 tactics during this study. We attempted to order the tactics by complexity according to the taxonomy outlined by Lee at al. (2008) in their study of human solvers. The mapping is inexact, as they describe clusters of similar tactics, rather than a strict order. However, the ordering we use for varying "knowledge" is approximately representative of the order in which humans generally acquire (or demonstrate) these tactics during actual puzzle-solving. This ordering also acts as a proxy for encoding complexity and cost.

We did not attempt to identify a complete set of tactics. The existing set of tactics is intended to reflect the first-cut, rapidly-encoded knowledge available during early development of a KE-engineered application.

\subsection{Deep Learning Solver}

Design decisions regarding the input feature representation, the neural network architecture, the output representation, and the output interpretation are required to satisfy the problem and use case of the application. 
Defining input and output representations encodes semantic information for use in the underlying model; these decisions are often domain-dependent ("feature engineering"). The selection of a specific neural network architecture is often dependent upon the domain, which dictates the types of layers (e.g., convolution layers used for image recognition) while the specific configuration - including the depth of the network or width of the layers - is determined using a parameter optimization process. The increased depth of layers — characteristic of a DL model - extract higher-level features and patterns upon which later layers may reason. Thus the complexity of the problem often dictates the number of layers required.

For the Sudoku task, we present the puzzle to the model in its entirety and receive weightings for particular cell values as output. Similar to the KE approach, we did not attempt to build a complete solver, but rather implemented a limited/naive solution that reflects the limited engineering time available in application development. This approach was sufficient to allow the KE and DL components to work together to solve a Sudoku.

\subsubsection{Input Representation}

Sudoku requires a categorical, not nominal, representation of cell values. To represent the categorical nature of inputs, we transformed each integer into a one-hot encoding for the labels of 'unknown' and the nine possible cell values. This representation generates an input tensor shape of $(9,9,10)$ in which a binary array of length 10 represents each cell.

\subsubsection{Output Representation and Interpretation}

Similar to the input representation, we encoded the output representation as a categorical output vector. While there are ten possible inputs, the outputs of the network should not include the 'unknown' category, and thus the output has a shape of $(9,9,9)$ with a $[0,1)$ range for each potential cell value $[1,9]$.

For each cell, the nine-valued vector is interpreted to be a probability weighting recommending a specific label. Rather than fill in all incomplete cells at once, we instead identity the cell with the the highest weighted label across all unsolved cells, a method recommended in (Park 2018). With that cell completed or filled in, we then present this Sudoku back to the neural network before inferring the next unsolved cell, continuing the process until the entire puzzle is complete.

\subsubsection{Neural Network Architecture}

We implement two functional layer groupings in the model, a feature extraction model followed by classifier model. To implement the feature extraction model, a fully connected component both takes in the input features and has several fully-connected layers as outlined in Table 2. These layers act as a feature extractor to identify interdependence between different input locations (LeCun, Bengio, and Hinton 2015). We use two fully-connected dense layers with sigmoidal activation functions followed by Dropout layers which act to prevent overfitting to the data (Srivastava, Hinton, Krizhevsky, Sutskever, and Salakhutdinov 2014). This output, representative of discovered computational features, is then flattened into a single one-dimensional vector of size 5184 .

This flattened output, comprising Sudoku "features", are input into a fully-connected dense categorical classifier model using a softmax activation function. Properties of this second classification layer are listed in Table 3. The softmax function allows the layer to predict a $[0,1]$ probability that a specific integer should be used to complete a particular space on the game board. Given both that there are 81 such classifiers, 
Table 2: Feature Extraction Model.

\begin{tabular}{ccc}
$\begin{array}{c}\text { Layer } \\
\text { (type) }\end{array}$ & $\begin{array}{c}\text { Output } \\
\text { Shape }\end{array}$ & $\begin{array}{c}\text { Parameter } \\
\text { Count }\end{array}$ \\
\hline \hline input_1 (Inputlayer) & (None, 9, 9, 10) & 0 \\
\hline dense_1 (Dense) & (None, 9, 9, 64) & 704 \\
\hline dropout_1 (Dropout) & (None, 9, 9, 64) & 0 \\
\hline dense_2 (Dense) & (None, 9, 9, 64) & 4160 \\
\hline dropout_2 (Dropout) & (None, 9, 9, 64) & 0 \\
\hline flatten_1 (Flatten) & (None, 5184) & 0 \\
\hline
\end{tabular}

Table 3: Softmax Cell Classifier Model.

\begin{tabular}{ccc}
$\begin{array}{c}\text { Layer } \\
\text { (type) }\end{array}$ & $\begin{array}{c}\text { Output } \\
\text { Shape }\end{array}$ & $\begin{array}{c}\text { Parameter } \\
\text { Count }\end{array}$ \\
\hline \hline $\begin{array}{c}\text { SeqFeature } \\
\text { Extractor (Flatten) }\end{array}$ & (None, 5184) & 0 \\
\hline Cell_n_dense (Dense) & (None, 9, 9, 64) & 46665 \\
\hline
\end{tabular}

one for each cell in the puzzle, and the parameters of the single feature extractor component, we must tune $3,784,729$ parameters during training.

\subsubsection{Training Process}

Training is conducted as a supervised learning process. Known inputs and expected output pairs are presented to the network. The difference between expected and actual outputs are used to adjust model weights in the direction that reduces difference. The goal of the learning is characterized by a loss function that must be minimized as more pairs are presented and processed.

Our incremental training strategy is to present progressively more difficult puzzles from training data. To accomplish this, we train by first presenting a complete, valid puzzle and then removing some number of completed cells (resulting in blanks). We then train the model by presenting a progressively increasing number of blanks within the puzzle.

The impact of this incremental approach was about 2-fold over a non-incremental approach. The incremental approach represents a (weak) form of transfer learning. By pre-training the model on a simpler puzzle, the model can incrementally fit the search space before trying to optimize for a more complex puzzle. Each training pass uses Adam optimization with categorical cross-entropy as the loss function measure to minimize (Kingma and Ba 2014).

During initial training, validation loss continues to decrease after several epochs. After several passes have occurred the number of epochs before over-fitting decreases as can be seen in Figure 2. This is due a decreasing amount of new information introduced into the network during subsequent training passes as the model has learned features from previous passes. The number of epochs is dynamic via the use of early stopping criteria that ends training after validation loss has not decreased after five Epochs. We perform iterative training passes until the model is operating on Sudoku puzzles with 64 blanks, or conversely puzzles with 17 clues, which form a lower bound on the number of possible clues (Delahaye 2006).

Training was conducted using a data set of various difficulty Sudoku puzzles with 25000 training instances, 7000 test instances and 3000 validation examples. Training examples were used to fit the neural network 

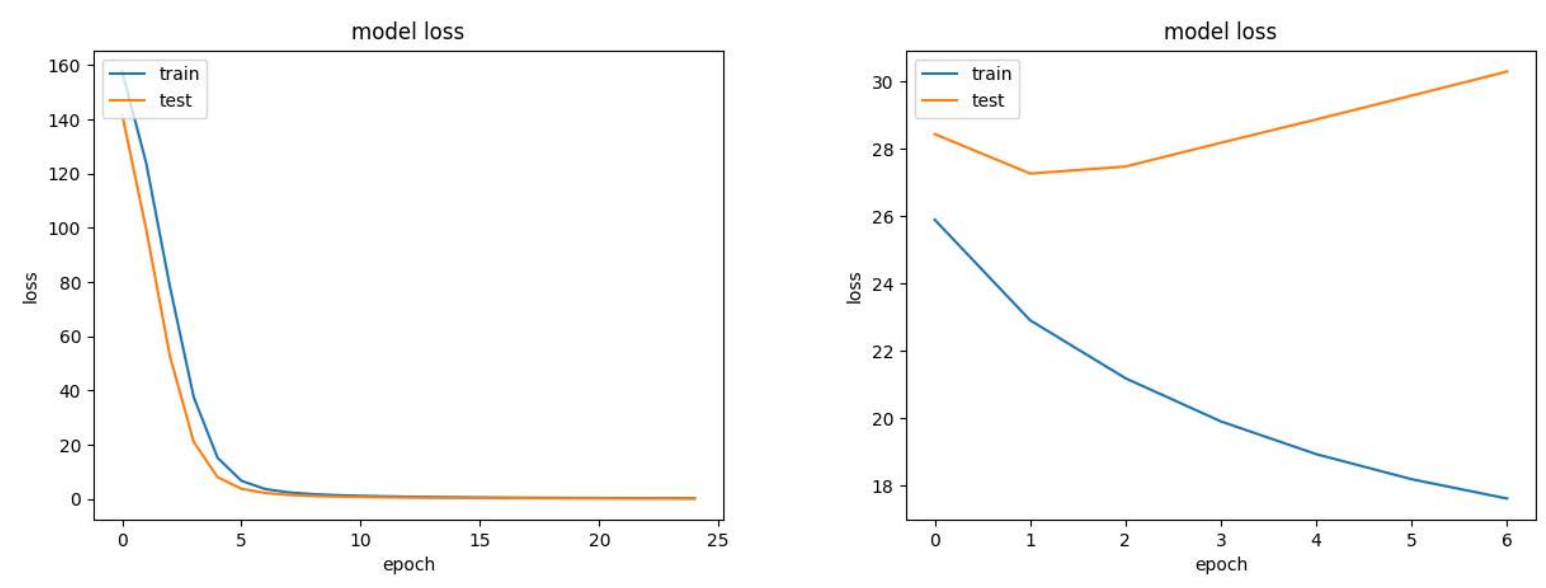

Figure 2: Training Pass 1 (Left) vs 40 (Right) Loss.

with the validation set acting to characterize generalization and overfitting. We collected performance metrics on all test examples. 35000 puzzles is an very small sample of the the total Sudoku problem space as described earlier.

\subsection{Hybrid KE/DL Approaches}

While different complex hybrid models are possible, we focused our exploration on two straightforward integrations. The first ("Sequential Hybrid Solver") represents the simplest possible combination of approaches. This method applies the KE tactics until it can make no additional progress toward a solution. Only then does it apply the DL approach (which will produce a single cell completion with the cell and value of highest confidence). The sequential solver iteratively queries the DL solver until no additional blanks remain on the puzzle. The sequential hybrid approach uses KE tactics to present a simpler Sudoku problem to the DL model.

The second approach is slightly more involved and uses KE tactics more interactively than the Sequential Hybrid Solver. This "Iterative Hybrid Solver' solver applies KE tactics after each DL inference (and keeps applying them until the puzzle is completed or no additional tactics can be applied). As a consequence, this approach uses the DL solver only when the KE tactics reach the limit of their effectiveness; The DL solver's effective role is to attempt to bring the problem back into a space where KE tactics are applicable.

\section{RESULTS}

This section outlines results from the point of view of the individual methods outlined in the previous section, both individually and in the two hybrid integrations we explored. For all results, we evaluate solver performance by reported the average proportion of correctly filled-in blanks within a puzzle by the given solver (normalized by the number of initial blanks). Thus, bounds of performance are [0,1] and 100\% performance would indicate all cells were completed correctly (the performance metric does not distinguish between cells left blank and cells filled incorrectly). All performance plots include a baseline representation for random guessing to allow comparison to the various methods. 


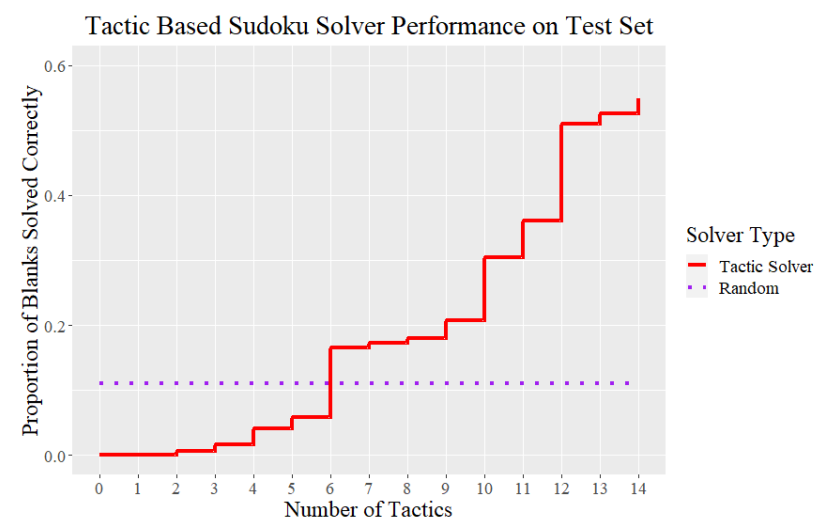

Figure 3: Tactics-based Solver Performance.

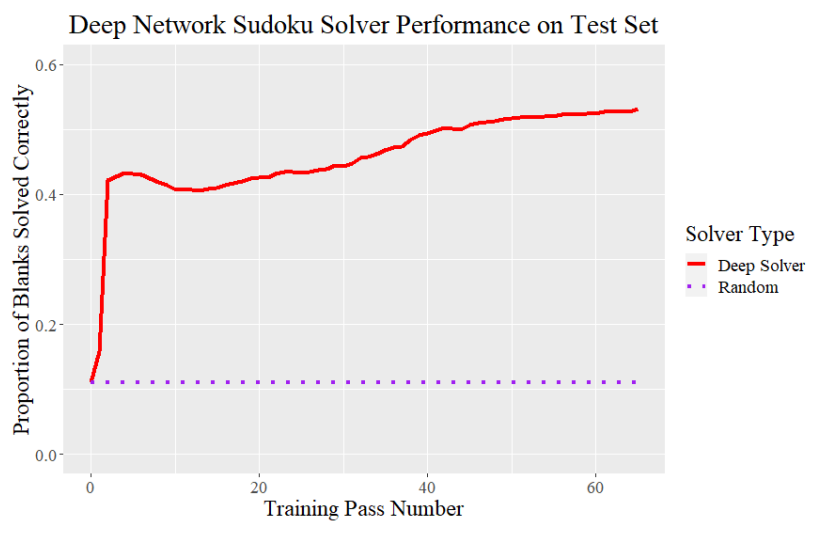

Figure 4: Deep Solver Performance.

\subsection{Tactics-based Solver Performance}

Figure 3 shows the performance results from the tactics-based solver. As expected, overall performance increased as additional tactics were made available to the tactics-based solver.

Looking more closely, the results suggest that the addition of some tactics have more significant impact on results. This effect derives from the general properties (and limitations) of knowledge-based solutions. The tactics-based solver operates by excluding candidates from cells. It it will not fill in a blank cell until there is only one candidate for that cell. When knowledge is added that allows a new pattern of common candidate eliminations, a large jump in performance can be observed.

Consider the addition of tactics 6 and 12, which are summarized in Table 1. Tactic 6 (Single Pairs) is the first tactic that considers constraints in the $3 \times 3$ sub-square, effectively combining row, column and box constraints for the first time. Tactic 12 (Hidden Pairs) considers $3 \times 3$ sub-squares, indicating the intersection of all three constraint dimensions. These tactics, which both consider the reductive power of "pairs" of candidates, are critical tipping points for hypothesis reduction and thus have an out-sized effect on performance when they are introduced.

The puzzle data-set was not fully solved. The tactics were sufficient to complete a little more than half of the blank cells in new puzzles (on average). We did not attempt to find and implement a complete set of tactics, as outlined previously. This limitation is reflective of the typical development of knowledge-based systems, where as the knowledge-based grows and becomes more complex, the incremental addition of knowledge becomes more costly.

\subsection{Deep-learning Solver Performance}

Figure 4 illustrates the results from the deep-learning solver alone as more training passes were completed with respect to performance on the test puzzle set. The performance associated with the DL solver follows the typical pattern of neural-network approaches: performance initially increases rapidly and then more slowly and incrementally improves with additional training. Recall that each training pass is representative of a variable number of Epochs of training with early stopping criteria and earlier training passes resulted in more training Epochs, as can be seen in Figure 2. This variable number of training epochs per pass were generally higher during earlier passes, explaining the sudden jump in performance during early passes with more gradual performance increases in later training passes. 


\section{Sudoku Solvers Performance Comparison on Test Set}

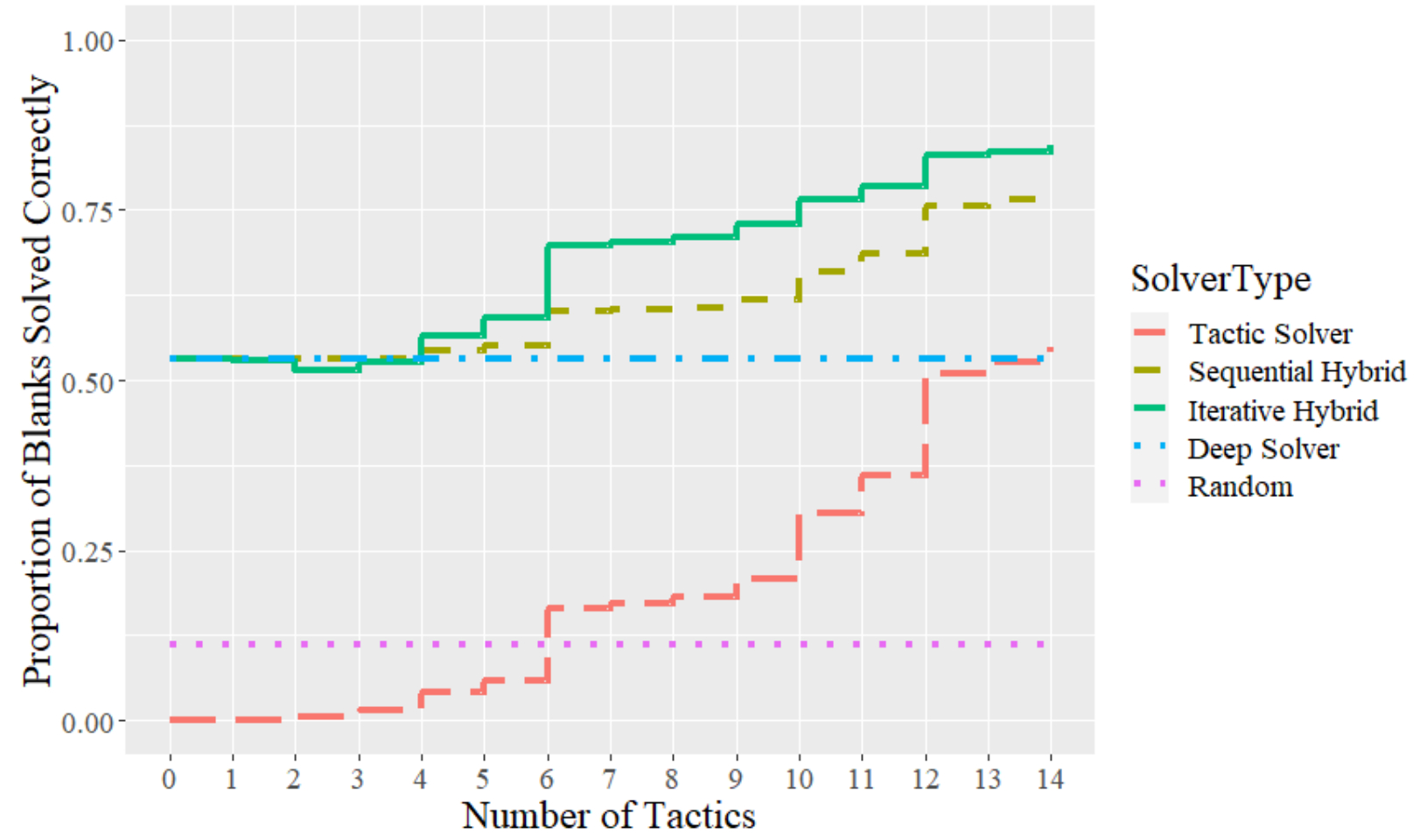

Figure 5: Solver Comparative Performance.

The DL solver on its own could only complete about half the blank cells, which is on par with the KE tactics solver. More exploration is required to determine if the very slow improvement was due to the complexity of the model or the relative sparsity of training data (35000 vs the $5.5 B$ essentially different completed grids (Russell and Jarvis 2006)).

\subsection{Hybrid Approaches}

Having looked at results from the individual techniques, we now examine results from their combined or hybrid use as summarized in Figure 5. The figure shows results from the two hybrid approaches introduced earlier, the tactics solver alone, the fully trained Deep Solver, and random guessing.

In the hybrid approaches, the tactics-based solver applies its rules prior to the use of the DL solver. Thus, the overall performance of both hybrid approaches is limited by the performance of the respective tactics-based solver. That is, as is clear in the figure, as the overall capability of the set of available tactics increases, including the significant steps in performance observed by the introduction of tactics 6 and 12, both hybrid approaches also demonstrate concomitant improvements in performance as well.

The iterative hybrid solver performs better than the sequential solver in all cases where the tactics-based solver implements more than two rules. Both hybrid solvers substantially outperform the tactics-based solver when the systems start with the same tactics. The better performance of the iterative solver derives directly from the iterative application of the KE and DL methods to individual puzzles. 
The DL solver was able to complete a bit more than $50 \%$ of cells in puzzles after 64 training passes (i.e., as in Figure 4). Both the hybrid solvers outperform the DL solvers. Conceptually, the sequential solver outperforms the DL solver by using the KE tactics to transform the problem into a simpler subspace problem that the DL solver is capable of solving itself. The iterative hybrid solver uses the DL solver to transform problems outside of the tactics-based solver solvable input space to the solvable set of problems.

\section{CONCLUSIONS}

We draw several conclusions from these results. First, while both the DL and the KE solvers were incomplete for solution of Sudoku, when combined, these two approaches provide greater performance and result in a more complete solution than either alone. The hybrid provides an ensemble of methods that improve overall performance in comparison to the individual algorithms.

Second, the results offer insights about knowledge-engineering requirements in addition to performance requirements, depending on development goals. If the goal of a system was to maximize performances, the hybrid methods increased solver performance by 53\% over the isolated KE tactics-based solver. However, if the goal was to realize an initial capability with little knowledge engineering, the hybrid methods reduce those requirements substantially as well. Assume a $70 \%$ correct completion average was a "reasonable" target for initial performance capability. This performance target is not reachable with only the 14 tactics we encoded. However, it is met with only 6 tactics (and only one "advanced" tactic) and the DL solver. Although more exploration is needed for a general conclusion, these results at least suggest that reasonably high levels of performance can be reached via a similar combination of knowledge and learning. KE methods can be used to encode common or base cases (usually the least expensive part of the process) and ML can then be used to rapidly explore the problem space and resolve special cases and edge conditions. Such edge cases are typically cost drivers in deployed knowledge-engineered systems.

Third, the tighter coupling in the Iterative Hybrid solution outperforms the Sequential Hybrid solution, suggesting that the level of interaction in the hybrid solution resulted in differences in performance. To date, we have focused on how the knowledge-engineering solution could be complemented by the DL solver (in part because we have developed multiple, deployed knowledge bases that might be enhanced in the future by ML hybridization). However, another direction for future exploration is to investigate the relative costs and benefits of further integration of the methods.

As one example, the DL solver described in this paper is trained without reference to or direct use of the tactics-based solver. However, it would be straightforward to target training of the DL solver to complete a cell whenever the tactics could no longer advance a solution. This tighter integration would further constrain and direct machine learning and potentially result in improved performance over even the hybrid methods presented here. Even deeper integrations can also be envisioned, such as using outputs of the DL solver (probabilistically-ranked potential completions) as inputs or triggers for the tactics.

For specific applications, these more sophisticated integrations may be warranted and beneficial in terms of the overall goals of a future system. However, more complex integrations make the technique more driven by the requirements of a particular domain and the expertise of the solution developers. For the purposes of this work, we achieved the goal of demonstrating that existing KE and ML methods - applied without thorough knowledge analysis or in-depth feature engineering - provide synergistic performance benefits. While this method should be further explored in other domains and contexts, the results suggest that significant gains can be achieved without complex and detailed engineering and integration between these seemingly disparate AI techniques. 


\section{REFERENCES}

Darwiche, A. 2018, September. "Human-level Intelligence or Animal-like Abilities?". CACM vol. 61 (10), pp. 56-67.

Delahaye, J.-P. 2006. “The Science behind SUDOKU”. Scientific American vol. 294 (6), pp. 80-87.

Feigenbaum, E. A. 1984. "Knowledge Engineering: The Applied Side of Artificial Intelligence". In Proc. of Symposium on Computer Culture: The Scientific, Intellectual, and Social Impact of the Computer, pp. 91-107. New York, NY, USA, New York Academy of Sciences.

Felgenhauer, B., and F. Jarvis. 2006. "Mathematics of sudoku I". Mathematical Spectrum vol. 39 (1), pp. $15-22$.

Kingma, D. P., and J. Ba. 2014. "Adam: A method for stochastic optimization". arXiv preprint arXiv:1412.6980.

LeCun, Y., Y. Bengio, and G. Hinton. 2015. “Deep learning”. Nature vol. 521 (7553), pp. 436.

Lee, N. Y. L., G. P. Goodwin, and P. N. Johnson-Laird. 2008. "The psychological puzzle of Sudoku". Thinking \& Reasoning vol. 14 (4), pp. 342-364.

Lynce, I., and J. Ouaknine. 2006. "Sudoku as a SAT Problem.”. In ISAIM.

Mikolov, T., I. Sutskever, K. Chen, G. S. Corrado, and J. Dean. 2013. "Distributed Representations of Words and Phrases and their Compositionality". In Advances in Neural Information Processing Systems 26, edited by C. J. C. Burges, L. Bottou, M. Welling, Z. Ghahramani, and K. Q. Weinberger, pp. 31113119. Curran Associates, Inc.

Mnih, V., K. Kavukcuoglu, D. Silver, A. A. Rusu, J. Veness, M. G. Bellemare, A. Graves, M. Riedmiller, A. K. Fidjeland, G. Ostrovski, S. Petersen, C. Beattie, A. Sadik, I. Antonoglou, H. King, D. Kumaran, D. Wierstra, S. Legg, and D. Hassabis. 2015, Feb. "Human-level control through deep reinforcement learning". Nature vol. 518 (7540), pp. 529-533.

Park, Kyubyong 2018. "Can Convolutional Neural Networks Crack Sudoku Puzzles?”. https://github.com/ Kyubyong/sudoku.

Russell, E., and F. Jarvis. 2006. "Mathematics of sudoku II". Mathematical Spectrum vol. 39 (2), pp. 54-58.

Schreiber, A. T., G. Schreiber, H. Akkermans, A. Anjewierden, N. Shadbolt, R. de Hoog, W. Van de Velde, N. R. Shadbolt, and B. Wielinga. 2000. Knowledge engineering and management: the CommonKADS methodology. MIT Press.

Srivastava, N., G. Hinton, A. Krizhevsky, I. Sutskever, and R. Salakhutdinov. 2014. "Dropout: a simple way to prevent neural networks from overfitting". The Journal of Machine Learning Research vol. 15 (1), pp. 1929-1958.

Strubell, E., A. Ganesh, and A. McCallum. 2019, Jun. "Energy and Policy Considerations for Deep Learning in NLP”. arXiv e-prints, pp. arXiv:1906.02243.

Sun, Y., A. Cuesta-Infante, and K. Veeramachaneni. 2019. "Learning Vine Copula Models for Synthetic Data Generation". In Proceedings of the AAAI Conference on Artificial Intelligence, Volume 33, pp. 5049-5057.

Wolfman, S. A., and D. S. Weld. 2001. "Combining linear programming and satisfiability solving for resource planning”. The Knowledge Engineering Review vol. 16 (1), pp. 85-99. 


\section{AUTHOR BIOGRAPHIES}

JOSHUA HALEY has a Master Degree in Computer Engineering with a Focus on Intelligent Systems and Machine Learning at the University of Central Florida. His expertise is in designing and using Machine Learning Systems for DoD use cases that are traditionally data constrained (cite). He is currently a Doctoral student at the University of Central Florida in the Modeling and Simulation program. joshua.haley@soartech.com

DR. ROSS HOEHN is a research scientist in Soar Technology's Intelligent Systems division. He earned is Ph.D. in Theoretical Chemistry from Purdue University in 2014, and continued research in chemical physics, quantum information and artificial intelligence until 2018. His research areas include: adaptive artificial intelligence, generative AI techniques, team learning and training, pedagogy, biological-based agent simulations, swarm mechanics, quantum information science, quantum computing and quantum mechanicallydriven biophysical phenomenon. He was an active researcher and manager of the NSF Center for Chemical Innovation: Quantum Information for Quantum Chemistry, a multi-million-dollar multi-year research effort centered at Purdue University to utilize quantum computing for quantum mechanical calculations. ross.hoehn@soartech.com .

DR. ROBERT WRAY received a Ph.D. in computer science and engineering from the University of Michigan. His doctoral research focused on maintaining logical consistency in agent reasoning systems and his innovations were incorporated in the Soar architecture. At Soar Technology, he leads or has led R\&D projects for the Air Force and Navy research offices and the Defense Advanced Research Projects Agency. Dr. Wray's research encompasses many areas of artificial intelligence, including agent-based systems and agent architectures, pedagogical applications of AI, machine learning, cognitive science and knowledge representation and ontology. Dr. Wray has become expert at bringing existing technology together with cognitivescience-inspired innovation to deliver solutions within the constraints of real problems. wray@ soartech.com 